\title{
Josse Bade, éditeur et préfacier $(1462-1535)^{*}$
}

\author{
MAURICE LEBEL
}

$\mathrm{J}$ osse Bade occupe un haut rang parmi les éditeurs-imprimeurs et les préfaciers de la Renaissance française. Ce grand humaniste, né en Belgique en 1462 et mort à Paris à la fin de 1535, vécut environ 47 ans en France, notamment à Valence et à Lyon, de 1488 à 1498, puis à Paris, de 1499 à 1535. Au lieu de signer son nom Josse Bade, à la française, ce qu'il lui arriva cinq fois seulement de faire dans sa vie, il préférait, à l'instar de la plupart des humanistes de son temps, le nom latin de Jodocus Badius, qu'il faisait ordinairement suivre de l'adjectif Ascensius, qui désigne soit la famille Van Asche de Gand ou la ville d'Ash, près de Bruxelles, où il naquit. Après avoir terminé ses humanités au collège de Gand, il alla suivre des cours de littératures grecque et latine aux universités de Ferrare et de Mantoue ${ }^{1}$ en Italie où il séjourna plusieurs années, probablement de 1480 à 1488; ce qui lui permit de connaître, par exemple, Ange Politien, Alde Manuce et Baptiste de Mantoue, et de voir le progrès de l'humanisme. On le voit ensuite enseigner la langue et la littérature latines à Valence et à Lyon. ${ }^{2}$ C'est dans cette dernière ville que, tout en enseignant le latin, il devint correcteur d'épreuves chez Jean Trechsel, imprimeur originaire d'Allemagne, dont il épousa la fille; de cette union devaient naître deux fils, qui furent de précieux collaborateurs, ${ }^{3}$ et six filles, dont quatre eurent pour époux de grands imprimeurs parisiens: Michel Vascosan, Jean de Roigny, Robert $I^{\text {er }}$ Estienne, Jacques $I^{\text {er }}$ du Pais.

C'est à Lyon même, en 1492, qu'il commença à publier, outre ses ouvrages personnels, des textes d'auteurs anciens et contemporains. En 1498 ou $1499,{ }^{4}$ peu après la mort de son beau-père, il s'installa à Paris, d'abord rue des Carmes, puis rue saint-Jacques, en plein quartier des collèges, des écoles et de la Sorbonne: c'est de son officine de publication, connue sous le nom de Praelum Ascensianum, que sortirent 708 éditions, de 1503 à 1535. Sans compter un nombre impressionnant de réimpressions, comme on le verra par la suite. Josse Bade appartient donc au

* Communication donnée le 29 mai 1980 lors d'une séance de travail de la société Canadienne d'Etudes de la Renaissance tenue a l'Université de Québec a Montréal. 
dernier tiers du $\mathrm{XV}^{\mathrm{e}}$ siècle par ses années de formation secondaire et universitaire et ses débuts dans l'enseignement et l'édition-imprimerie, comme il appartient au premier tiers du XVI ${ }^{\mathrm{e}}$ siècle par son immense production, qui reste unique dans les annales de l'imprimerie en France. Elle s'échelonne sur une période de 43 ans, notamment à Lyon et à Paris, de 1492 à 1535. Sa vie active s'écoula sous les règnes de Charles VIII (1483-1498), de Louis XII (1498-1515), de François I ${ }^{\text {er }}$ (15151547 ) et de Charles-Quint (1500-1558), son compatriote. Il connut, outre les progrès de l'humanisme en Italie et en France, en Angleterre, en Allemagne et dans les Pays-Bas, les débuts de la Réforme et la plupart des humanistes de son temps. ${ }^{5}$

Josse Bade a publié une oeuvre si volumineuse, en un demi-siècle, qu'elle ne manque pas de provoquer l'admiration et l'étonnement. D'autant plus qu'il l'a menée à bonne fin, presque tout seul, avec l'aide de rares collaborateurs: il semble avoir vécu uniquement en vue de son imprimerie, sa raison d'être, à tel point sa foi était profonde dans le prestige de l'écrit et dans la puissance de la récente invention de Gutenberg. Aussi a-t-il laissé une oeuvre monumentale qui comprend une quinzaine de volumes de biographie et d'histoire, d'éducation et de morale, de philosophie et de théologie, 34 traités de grammaire, 72 commentaires d'auteurs et de textes latins, ${ }^{6} 103$ poèmes latins, dont les plus importants sont ceux de 1497, de 1501 et de 1505, qui comprennent respectivement 40,31 et 44 vers, $^{7} 226$ préfaces, 775 textes anciens et contemporains, enfin une vaste correspondance avec les humanistes les plus prestigieux de son temps, par exemple, avec Erasme, Budé, Jacques Toussaint et Jacques Lefèvre d'Etaples. Etant donné l'ampleur de cette production, je vais m'en tenir, dans cette communication, aux éditions et aux préfaces de Josse Bade.

\section{Editions}

Les éditions de Josse Bade comprennent trois catégories: celles de ses oeuvres personnelles, celles des auteurs contemporains, celles des auteurs grecs et latins, du Moyen Age et de la Renaissance. Il a vu lui-même de près à l'édition de ses propres volumes, comme à celle des auteurs anciens et des auteurs de son temps. On les distingue toutes assez facilement du premier coup d'oeil. A de très rares exceptions près, elles sont constamment datées d'après le style romain. Elles sont remarquables parl'exécution typographique, l'impression correcte et régulière: l'usage des caractères gothiques y est fréquent, surtout dans les ouvrages religieux. Quelques-unes sont précédées d'un important traité, par exemple, sur la comédie ou la tragédie, sur l'histoire ou l'art épistolaire; ces traités, souvent réimprimés, exercèrent une influence considérable sur le théâtre, sur la biographie et l'histoire, sur la correspondance des écrivains de son 
temps et sur l'imitation du style de Cicéron par beaucoup d'humanistes. Bon nombre sont aussi accompagnées de notes, de commentaires et de préfaces. La plupart des volumes, parus de 1492 à 1535 , sont des in-folio ou des in-quarto; très peu nombreux sont les in-octavo, ce petit format comprenant de courts traités de morale et de théologie, des sermons, quelques livres de médecine, des recueils de poésies d'auteurs contemporains, voire des classiques annotés comme Horace, Juvénal et Ovide. Innombrables aussi sont les contrefaçons des éditions imprimées par Badius, car la piraterie était alors d'usage courant dans l'imprimerie comme sur les mers; le droit d'auteur était encore inconnu à l'époque de la Renaissance. D'ailleurs, Josse Bade semble avoir été trop occupé par ailleurs pour entretenir un véritable souci de tirer le meilleur parti de ses oeuvres d'auteur-éditeur-imprimeur.

Ce qu'on remarque aussi dans cette immense production, c'est le très petit nombre de volumes sur vélin: j'en ai relevé au plus $7:{ }^{8}$ une édition avec des commentaires écossais, une autre avec des notes en flamand, et seulement quatre en français. ${ }^{9}$ On relève aussi, par exception, quelques éditions anonymes. ${ }^{10}$ L'année la plus productive de l'imprimerie fut sans doute 1519, qui vit paraître 39 éditions distinctes: la plus pauvre, avec une seule édition, fut 1525, par suite de la crise politique en France: 1504 et 1535 furent aussi deux années assez maigres à cet égard, puisque 7 et 6 éditions respectivement sortirent alors du Praelum Ascensianum de la rue Saint-Jacques. La production décennale s'établit de la façon suivante: de 1503 à 1512,212 ; de 1514 à 1524,264 ; de 1525 à 1535 , 180. Un total de 700 éditions, auxquelles il faut ajouter celles qui ont paru de 1492 à 1502, puis les éditions de grammaire, et quelques livres non datés avec précision, mais portant la marque de l'imprimerie de Badius. En tout, 775 éditions, de 1492 à 1535.

Si nous poursuivons quelque peu l'analyse descriptive de ces impressions, nous aurons tôt fait d'en relever les autres traits suivants. On remarque seulement quatre traductions françaises, l'histoire de Thucydide et celle de Diodore de Sicile, par Claude de Seyssel, la Vie de saint Jérôme par Louis Lasserre et la Vie de Jésus par Ludolphe de Saxe. Ce qui frappe aussi, outre la rareté des impressions de textes français, c'est celle des textes grecs. Fait étrange, c'est en 1520 seulement que Bade, sur la demande expresse de Budé et d'Erasme qui s'en plaignaient, a fait venir d'Allemagne une fonte de grec, ce qui lui permit d'entreprendre des impressions dans cette langue. L'Allemagne était en avance sur la France à cet égard, car Luther y avait déjà in troduit le grec dans l'université; Guillaume eut tôt fait de l'imiter en France. La même année, en 1520, Bade imprima un recueil de lettres grecques et latines de Guillaume Budé; il se servit alors des mêmes caractères élégants que ceux employés par Erasme pour le Nouveau Testament, publié à Bâle 
par Nicolas Episcopius en 1520, 1522 et 1531. Bade devait imprimer aussi, de Budé, les Commentarii linguae grecae en 1529. A vrai dire, le célèbre imprimeur parisien ne fit pas un fréquent usage du grec. Tout au plus imprima-t-il, outre les deux ouvrages de Budé déjà mentionnés, les textes suivants en grec: trois discours ou essais d'Isocrate: Éloge d'Hélène, Nicoclès, Aréopagitique (1529), les épigrammes de Janus Lascaris (1527, 1531), le traité d'Aristote et de Philon le Juif sur Le Monde (1526), un Florilège d'épigrammes diverses (1531), la grammaire grecque du grand helléniste de la Renaissance, Théodore Gaza (1521), l'essai de Démosthène, médecin grec du $\mathrm{I}^{\mathrm{er}}$ siècle de notre ère, Sur la vieillesse (1532).

L'extrême rareté des impressions des textes grecs forme un violent contraste avec la publication d'un très grand nombre de traductions latines de textes grecs: on peut même dire que c'est alors l'âge d'or de la traduction latine des oeuvres grecques. C'est que le grec, de son temps, et surtout avant 1520, en France du moins, était encore beaucoup moins connu que le latin, et cela, malgré les efforts de Guillaume Budé et de Jacques Lefèvre d'Etaples pour l'instaurer; il était alors beaucoup plus répandu en Allemagne et en Angleterre, en Italie, en Flandre et en Hollande, voire en Suisse. Guillaume Budé, il est vrai, travaillait ferme à le restaurer en France avec l'aide d'Erasme, de Jacques Toussaint et de Germain de Brie, non sans rencontrer une vive opposition de la Sorbonne, où les gendarmes étaient alors plus nombreux que les théologiens. De toute façon, Josse Bade imprima un très grand nombre de textes grecs en traduction latine, d'Homère à Eusèbe de Césarée; il imprima en latin la traduction des oeuvres d'Hérodote et de Thucydide, de Platon et de Théocrite, de Polybe et de Diodore de Sicile, d'Origène, d'Eusèbe et de Basile le Grand; il publia aussi, en partie, la traduction latine de quelques oeuvres d'Homère et d'Hippocrate, de Démosthène et d'Aristote, de Xénophon et de Flavius Josèphe, de Lucien et de Plutarque, de Philon le Juif, de Grégoire de Nysse, de Grégoire de Nazianze et de Jean Chrysostome. Ces traductions latines connurent un grand succès au XVI ${ }^{\mathrm{e}}$ siècle et furent souvent réimprimées.

Mais ce sont les Latins qui furent des auteurs à succès du temps de Josse Bade et au XVI $\mathrm{XI}^{\mathrm{e}}$ siècle. Voici une courte liste des éditions des bestsellers latins au XVI ${ }^{e}$ siècle: Macrobe (2), Sénèque le tragique (2), Valérius Flaccus (3), Tite-Live (3), Cicéron (4), Lucain (4), Aulu-Gelle (4), Quintilien (6), Ausone (7), Juvénal (17), Valère Maxime (20), Perse (21), Horace (24), Salluste (25), Virgile (27), Boèce (29), Ovide (35) et Térence (49). A noter le goût prononcé du public pour les poètes de préférence aux prosateurs. Innombrables aussi furent les impressions et les réimpressions des discours et des traités de philosophie surtout de 1527 à 1534; le cicéronianisme est alors à la mode parmi les érudits. 
D'autre part, Josse Bade imprima aussi bon nombre d'oeuvres particulières d'écrivains de la littérature chrétienne tels que: Ambroise, Augustin, Benoît, Bède, Bruno, Grégoire de Tours, Hilaire, Lactance, Paulin, sans oublier Antoine de Padoue, Bonaventure, Duns Scot, Thomas d'Aquin et Thomas à Kempis. De ce dernier, Josse Bade a aussi écrit la biographie. Fait digne de remarque, il fait ordinairement précéder l'édition du texte d'une courte biographie de l'auteur imprimé.

Loin de s'intéresser uniquement aux auteurs grecs et latins de l'Antiquité païenne et chrétienne, du Moyen Age et du début de la Renaissance en Italie, Josse Bade s'est aussi beaucoup intéressé aux auteurs des XVe et $\mathrm{XVI}^{\mathrm{e}}$ siècles, en somme à ses contemporains. Il a en effet imprimé les oeuvres de 155 d'entre eux, parmi lesquels figurent au premier rang: Erasme, Guillaume Budé, Jacques Lefèvre d'Etaples, Thomas More, John Fischer, Savonarole, Aldus Manutius, Janus Lescaris, Théodore Gaza, Claude de Seyssel, Louis Lasserre, Laurentius Valla, Germain de Brie, Jean Sulpitius, Marcilinus Ficinus, Nicolas de Cuse, Baptiste de Mantoue, Christophe de Longueuil, Angelus Politien, Philippe Béroalde L'aîné, Jacobus Perez de Valentia, Pierre Bury, les papes Léon X, Hadrien VI et Urbain IV. En bref, les oeuvres des contemporains occupent une place très importante dans l'ensemble des éditions-impressions de Josse Bade; bon nombre d'entre eux, je crois, ont été sauvés de l'oubli grâce à lui. Il a eu l'idée généreuse, en véritable érudit et imprimeur humaniste qu'il était, d'imprimer des textes d'auteurs relativement peu connus: il a montré aussi assez de flair pour imprimer des textes dont la vente était rentable. Grâce aux nombreux contacts qu'il entretenait, du haut de son officine de publication avec les humanistes de son temps, au pays et à l'étranger, laïques et religieux, il lui arriva plus d'une fois de répondre à des commandes spécifiques d'impression.

Ancien professeur d'humanités, éducateur-né et humaniste averti, il brûlait de répandre l'enseignement du latin et de communiquer son enthousiasme pour la renaissance des lettres anciennes, inséparable de son amour des lettres contemporaines. Et c'est ce qui fait sa profonde originalité. Il n'existe point pour lui de solution de continuité entre le passé et le présent, entre le Moyen Age et la Renaissance. Il s'intéresse tout ensemble aux anciens et aux modernes. Cette conviction profonde explique l'acharnement avec lequel il a édité et réédité, annoté et commenté des textes, des morceaux choisis, des sélections, des traités de grammaire, d'éducation et de morale. Il pensait toujours aux jeunes et à l'avenir. Il ne se contenta pas de restituer le passé, il travailla aussi à bâtir l'avenir. Au fond, directeur-propriétaire de la plus importante maison d'édition de son temps, il avait pleinement conscience de jouer un rôle et d'exercer une influence en son milieu. 


\section{Préfaces}

Ce sentiment personnel ressort aussi nettement des Préfaces, 226 bien comptées, qu'il écrivit, non seulement pour ses propres ouvrages, comme les Sylvae Morales et les Stultiferae Naves, mais aussi et surtout pour les oeuvres particulières des écrivains grecs et latins de l'Antiquité, du Moyen Age et de la Renaissance qu'il édita. Ces 226 préfaces servent d'annonce ou d'introduction à peu près au tiers des volumes qu'il imprima. Il s'agit donc d'une habitude assez constante chez lui. Ajoutons que 60 d'entre elles sont adressées tantôt à la jeunesse studieuse (26) et aux professeurs (15) de son pays et de l'étranger, tantôt à des libraires (15) et à des écrivains bien connus (6); ce sont les plus sérieuses de toutes. Il en est d'autres, environ 80, que l'auteur envoya aux gens d'église: prêtres, chanoines, évêques, archevêques, cardinaux, abbés et religieux de tous ordres; elles sont aussi importantes que celles qu'il composa pour Guillaume Budé, Louis Ruzé et Guillaume Petit, si influents auprès de François Ier. L'auteur avait raison de les écrire en si grand nombre pour se ménager les bonnes grâces des gens en place à qui il adressa des compliments hauts comme les Rocheuses; il fait de même à son égard, Corneille et Racine en feront autant au XVII ${ }^{e}$ siècle, la modestie n'étant pas la qualité fondamentale de la Renaissance. D'ailleurs, il fallait alors frapper fort, l'imprimerie étant surveillée par des censeurs sourcilleux. Aussi était-il de bonne guerre de compter de solides protecteurs au nombre de ses amis.

Toutes ces préfaces, Josse Bade les a rédigées dans le dessein bien avoué d'être lu et entendu. Il importe peu qu'il ait emprunté à l'occasion une phrase ou deux à des recueils de préfaces déjà composés à cet effet en Italie; ou qu'il ait demandé à un collaborateur de lui fournir desidées pour les écrire; l'important est qu'elles portent la marque de son style et qu'elles jettent un éclairage singulier sur l'homme, son milieu et son temps.

Du côté des écrivains grecs, méritent d'être lues les préfaces aux oeuvres d'Homère, d'Hérodote et de Thucydide, d'Aristote et de Platon, de Polybe et de Plutarque, de Flavius Josèphe et de Lucien, d'Origène et de saint Basile. Parmi les préfaces consacrées aux auteurs latins, je retiens surtout celles, fort étoffées, de Salluste et de Sénèque, de Térence et de Tite-Live, de Cicéron et de Virgile, de Juvénal et de Perse, de Quintilien et de Boèce, de Grégoire de Tours et de saint Augustin, de saint Bruno et de saint Antoine de Padoue. Il convient de ranger parmi les plus senties et les mieux tournées celles de saint Bruno, de Guillaume d'Ockam, de Pétrarque et de Laurent Valla, de Philippe Béroalde, de Baptiste de Mantoue et de Jacobus Perez de Valentia. Je compte publier un jour la traduction française de toutes ces préfaces que je viens de mentionner. 
Elles font ressortir le goût des contemporains pour la prose historique et oratoire, morale et philosophique, pour la poésie comique et tragique, pastorale et satirique, pour la mythologie et les questions religieuses. Elles nous renseignent sur les courants d'idées et les progrès de l'humanisme, les points de vue religieux et les attitudes sexuelles dans les livres d'éducation. Elles contiennent des opinions pédagogiques fort instructives, originales et hardies, constructives et pratiques, dont Port-Royal saura faire un excellent usage au XVII ${ }^{e}$ siècle. Josse Bade les écrivit régulièrement pendant 43 ans, de 1492 à 1535 , au rythme de 4 en 1496 , de 5 en 1492 et 1509 , de 6 en $1500,1502,1504,1512,1516,1520$, 1521,1524 , de 7 en 1501 et 1519 , de 8 en 1499 et 1528 , de 9 en 1506 et 1513 , de 10 en 1514 , de 12 en 1511 , de 14 en 1503. Peu d'auteurs contemporains du $\mathrm{XX}^{\mathrm{e}}$ siècle ont commis autant de préfaces durant leur carrière. Aucun autre éditeur-imprimeur du temps de Bade n'a produit autant de préfaces que lui. Comme bien l'on pense, elles ne sont pas toutes d'égale longueur ou d'égale importance.

Diverses sont les raisons pour lesquelles Josse Bade les compose. Tantôt ce fut pour plaire aux grands du jour et s'attirer leurs bonnes grâces, tantôt pour répandre ses idées personnelles et exprimer ses convictions profondes: il le fit aussi souvent pour présenter un auteur nouveau ou un ouvrage peu connu; il lui arriva également de répondre par anticipation aux critiques personnelles: on a le sentiment parfois qu'il met sa vanité à nu et qu'il se distribue des compliments à l'envi: plus d'une se lit comme un acte de civilité, voire comme un pensum écrit par l'auteur à son corps défendant. Quoi qu'il en soit, on pourrait en extraire une philosophie de la vie, une gerbe d'idées en matière d'éducation et de littérature, de morale et de religion, voire une certaine évolution dans la pensée religieuse de Josse Bade. A noter qu'il y parle beaucoup moins de lui-même que de ses collaborateurs et de ses assistants, des libraires et des imprimeurs.

Plusieurs préfaces connurent des réimpressions. Elles contribuèrent à la réputation du préfacier parmi les humanités de son temps et dans le grand public lettré de France et de l'étranger. Sans doute son latin, dans l'ensemble assez direct et simple, truffé qu'il est cependant de néologismes, de mots pittoresques, de tours ingénieux, d'expressions inconnues de Jules César et de Cicéron, est-il moins limpide et moins pur que celui d'Erasme, de Murat et de Christophe de Longueuil, les meilleurs latinistes $\mathrm{du} \mathrm{XVI} \mathrm{X}^{\mathrm{e}}$ siècle. Il n'empêche que Bade exerça une influence considérable sur l'enseignement du latin dans les collèges, non seulement par ses éditions et ses préfaces, mais aussi par ses commentaires familiers et ses traités de grammaire. On y étudiait les auteurs anciens, de même qu'on s'essayait à écrire un latin cicéronien. Bien plus, on y enseignait les auteurs vivants aussi bien que les écrivains de l'Antiquité. Ne fait-on pas 
de même aujourd'hui dans nos collèges et dans nos universités? La préface de ses Sylvae Morales, qui sont une anthologie des auteurs anciens et des auteurs contemporains, est instructive à cet égard, puisque l'auteur y explique les divers motifs qui doivent pousser les gens à l'étude du latin; on y trouve aussi d'excellentes méthodes de mémorisation, d'analyse et de commentaire de textes. Bien plus, il ne suffit pas d'enseigner la morale en mettant les défauts et les vices à nu; avec un sans-gêne total, on étalait alors, dans les textes, au XVI ${ }^{\mathrm{e}}$ siècle, les vices dans toute leur horreur pour en inspirer le profond dégoût; il faut enseigner aussi la belle langue, la langue châtiée, le beau et le bon latin. On ne disait pas encore le beau et le bon français. Mais cela devait venir peu après.

Il n'est certes pas facile de mesurer, en conclusion, l'impact des éditions-impressions et des préfaces de Josse Bade sur son milieu, sur son temps et sur les générations subséquentes au XVI ${ }^{\mathrm{e}}$ siècle. Dans quelle mesure il contribua ainsi à répandre l'humanisme, à atteindre un grand public cultivé, à promouvoir l'étude de l'Antiquité et de la Renaissance, à perfectionner les méthodes d'étude et d'enseignement du latin, à faire connaître les oeuvres de bon nombre d'humanistes de son pays et de l'étranger, il n'est pas non plus aisé de le prouver exactement. Ce qu'on sait de certitude, c'est qu'il avait confiance en lui-même et qu'il avait conscience de faire une oeuvre utile en imprimant et en réimprimant quantité de textes anciens et contemporains. Sans doute appartenait-il au monde des érudits et des humanistes pour qui le latin était encore à cette époque la langue internationale de communication. A l'instar de Guillaume Budé, il ne fit pas un grand usage du français dans ses oeuvres. Vivant quelque peu à l'écart ou en marge des écrivains d'imagination, qui utilisaient de plus en plus le français dans leurs écrits, comme François $I^{\text {er }}$ qui ne comprenuit pas le latin, Josse Bade s'exprimait en latin, comme le faisaient presque tous les érudits de son temps. Bien plus, son intérêt était vaste, car il s'intéressait au droit, à la philosophie, à la théologie, à la philologie, à la grammaire et à la littérature. Il a fini par imposer sa propre grammaire et par se débarrasser, avec raison, de celle d'Alexandre de Villadieu, qui était en usage depuis le Moyen Age. A en juger du moins par le très grand nombre des impressions et réimpressions sorties de son officine de publication, à en juger aussi par le grand nombre des contrefaçons que libraires et éditeurs firent de son oeuvre au XVI siècle, ce dont il se plaignait amèrement, il a accompli un travail utile et fécond. Non seulement il a fait connaître les oeuvres de bon nombre d'érudits et d'humanistes français en France et à l'étranger, mais il a aussi imprimé et répandu les oeuvres de quantité d'humanistes étrangers dans les pays qui forment aujourd'hui la Grande-Bretagne, l'Allemagne, la Belgique, la Hollande et l'Italie. La Renaissance des arts et des lettres, 
commencée en Italie, eut tôt fait de se répandre à l'étranger. L'oeuvre monumentale de Josse Bade contribua énormément à cet heureux résultat.

\section{Université Laval}

\section{Notes}

1 Il suivit Ies leçons du savant lexicographe Giovanni-Battista Guarini (1425-1513). Mantoue possédait alors une université.

2 Il y avait aussi une université à Valence. A Lyon, il enseigna une dizaine d'années au collège dirigé par Henri Valluphinus; il resta fidèle et reconnaissant au recteur du collège, comme le révèle sa préface aux Satires de Juvénal (1498), qu'il lui adressa; c'est au même chanoine qu'il adressa deux autres préfaces, tout à fait différentes, en 1499, pour le Parthenice Catharinaria de Baptiste de Mantoue, et en 1506, pour les Satires de Juvénal.

3 L'aîné mourut subitement en 1526. Conrad assura la succession de l'imprimerie en 1536, peu après la mort de son père en 1535. Mentionnons, parmi les collaborateurs ou les correcteurs, outre ses deux fils, les personnes suivantes; Louis Fidèle, Michel Pontanus, Beatus Rhenanus, Jean Louys de Thielt, Georges Scanfelaer de Gand, Louis Blanblonc de Gand.

$4 \mathrm{La}$ biographie de Josse Bade reste à faire. Il nous manque encore beaucoup de renseignements précis sur sa vie, sur ses idées pédagogiques, politiques et religieuses.

5 Guillaume Budé, Erasme, Jacques Lefèvre d'Etaples, Jacques Toussaint, Germain de Brie, Laurent Bureau, Jean Petit, Guillaume Petit, Beatus Rhenanus, Louis de Berquin, Jean London, Pierre Danès, Louis Lasserre, Guillaume du Maine, et alii.

6 Je n'ai relevé aucun commentaire de textes grecs, bien qu'il ait publié une dizaine d'auteurs grecs. Les commentaires familiers de Bade remportèrent un vif succès auprès des étudiants et des professeurs au $\mathrm{XVI}^{\mathrm{e}}$ siècle.

$7 \mathrm{La}$ plupart des poèmes latins de Badius sont de courtes pièces volantes, écrites pour les volumes que l'imprimeur publiait. 1497: De praestantia Gallorum et auctoris (40 vers) 1501: De Insignibus Francine (31 vers)

1505: De Annunciatione dominica (44 vers)

8 Oeuvres d'Origène (1512); Argonautiques de Valérius Flaccus (1517-1519): Timée de Platon (1520); Thucydide, trad.fr. (1527); Budé, Commentarii linguae grecae (1529); Budé, De Studio Litterarum (1532); Budé, De Philologia (1532).

9 L'Histoire de la guerre du Péloponnèse (1527) par Thucydide et l'Histoire des successeurs d'Alexandre (1530) par Diodore de Sicile, toutes deux traduites par Claude de Seyssel. Ludolphe de Saxe traduisit la Vie de Jésus (1502) et Louis Lasserre, La Vie de saint Jérôme (1529).

10 Biblia aurea (1510); Dogma moralium philosophorum (1511); Annotationes doctorum virorum (1511); Grammatici illustres duodecim (1516), Remedia septem contra amorem illicitum (1521); Annotationes virorum illustrium in quaedam Ciceronis loce (1528). 